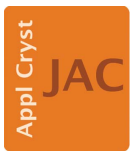

JOURNAL OF APPLIED CRYSTALLOGRAPHY

ISSN 1600-5767
Received 15 September 2017

Accepted 15 September 2017

Keywords: modulated diffuse scattering; local structure modeling; Lorentz and spectrum corrections; single-crystal time-of-flight neutron diffraction

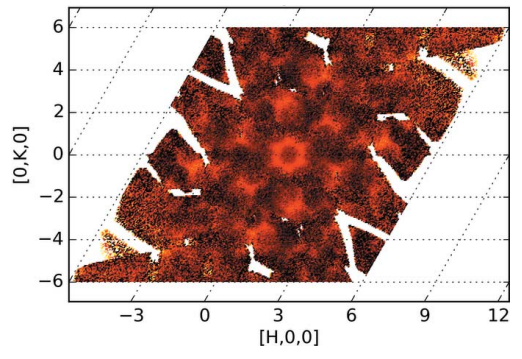

OPEN $\odot$ ACCESS

\section{Expanding Lorentz and spectrum corrections to large volumes of reciprocal space for single-crystal time-of-flight neutron diffraction. Corrigendum}

Tara M. Michels-Clark, ${ }^{a}$ Andrei T. Savici, ${ }^{b}$ Vickie E. Lynch, ${ }^{\text {b }}$ Xiaoping Wang, Michal Chodkiewicz, ${ }^{\text {a,c }}$ Thomas Weber, ${ }^{d}$ Hans-Beat Bürgi ${ }^{d, e}$ and Christina M. Hoffmann ${ }^{\mathbf{b}}$ *

a'Lawrence Berkeley National Laboratory, Berkeley, CA 94720, USA, ${ }^{\mathbf{b}}$ Neutron Sciences Directorate, ORNL, PO Box 2008 - MS 6475, Oak Ridge, TN 37831-6475, USA, 'Department of Chemistry, Warsaw University, Pasteura 1, Warsaw 02-093, Poland, 'Laboratory of Crystallography, ETH Zürich, Wolfgang-Pauli-Strasse 10, HCl G505, CH-8093 Zürich, Switzerland, and ' Department of Chemistry and Biochemistry, Universität Bern, Freiestrasse 3, CH-3012 Bern, Switzerland. *Correspondence e-mail: choffmann@ornl.gov

The author list of the article by Michels-Clark et al. [J. Appl. Cryst. (2016), 49, 497-506] is amended with the addition of Michal Chodkiewicz, Thomas Weber and Hans-Beat Bürgi. The complete list of authors is Tara Michels-Clark, Andrei Savici, Vickie Lynch, Xiaoping Wang, Michal Chodkiewicz, Thomas Weber, Hans-Beat Bürgi and Christina Hoffmann.

The author list of the publication by Michels-Clark et al. (2016) is amended, with Michal Chodkiewicz, Warsaw University, Thomas Weber, ETH Zürich, and Hans-Beat Bürgi, Universities of Bern and Zürich, as full-fledged coauthors. Tara Michels-Clark, Andrei Savici, Vickie Lynch, Xiaoping Wang and Christina Hoffmann apologize for the omissions.

The contributions of the individual authors to the article are as follows:

Tara Michels-Clark, Vickie Lynch, Andrei Savici, Xiaoping Wang and Christina Hoffmann prepared the manuscript, compared different correction algorithms for the diffuse scattering neutron diffraction data, and analyzed and developed the data processing workflow. The mathematical treatment of the Lorentz-correction and statistical weighting scheme was devised by Andrei Savici and Tara Michels-Clark.

The neutron data were obtained in a joint experiment between the ORNL and Zürich groups. Neutron single-crystal diffraction data from the SNS TOPAZ diffractometer were collected and refined by Tara Michels-Clark, Christina Hoffmann and Xiaoping Wang. Complementary X-ray data at $100 \mathrm{~K}$ were measured and refined by Tara Michels-Clark and Christina Hoffmann. Tara Michels-Clark statistically compared the Bragg data obtained by different processing protocols.

Tara Michels-Clark analyzed the diffuse data using the Monte Carlo crystal builder and intensity calculations in ZODS. Michal Chodkiewicz, Thomas Weber and Hans-Beat Bürgi developed the $Z O D S$ software for model building and refinement. Hans-Beat Bürgi co-mentored Tara MichelsClark's PhD thesis with Christina Hoffmann.

\section{References}

Michels-Clark, T. M., Savici, A. T., Lynch, V. E., Wang, X. P. \& Hoffmann, C. M. (2016). J. Appl. Cryst. 49, 497-506. 